Recepción: 20 / 04 / 2017

Aceptación: 20 / 05 / 2017

Publicación: 15 / 06 / 2017

Ciencias de la Salud

Artículo de Investigación

\title{
Relación entre Obesidad y Diabetes Mellitus tipo II en Ecuador
}

\author{
Relationship between Obesity and Diabetes Mellitus type II in Ecuador
}

\section{Relação entre obesidade e diabetes mellitus tipo II no Equador}

M.Sc. Josefina E. Ramírez-Amaya ${ }^{\text {I }}$ josefina.ramireza@ug.edu.ec

M.Sc. Pedro L. Maldonado-Álava II pedro.maldonadoa@ug.edu.ec

M.Sc. Asdrubal M. Fabre-Parrales III asdrubal.fabrep@ug.edu.ec

M.Sc. Julio J. De la Torre-Chávez IV juliodelatorrec@hotmail.com

Correspondencia: josefina.ramireza@ug.edu.ec

Master en salud pública, Especialista en docencia superior, Doctora en medicina y cirugía. Docente Universidad de Guayaquil, Guayaquil, Ecuador.

II. Magister en Salud Pública, Diplomado en docencia superior, Doctor en medicina y cirugía. Docente Universidad de Guayaquil, Guayaquil, Ecuador.

III. Master en Docencia universitaria e investigación educativa, Especialista en patología clínica, Doctor en medicina y cirugía. Docente Universidad de Guayaquil, Guayaquil, Ecuador.

Iv. Master en Gerencia Educativa, Especialista en Gestión de Procesos Educativos, Diplomado en Docencia Superior, Docente Universidad de Guayaquil, Guayaquil, Ecuador. 


\section{Resumen}

El objetivo del presente trabajo es determinar la relación existente entre Obesidad y Diabetes Mellitus Tipo II y describir las causas que produce la obesidad. las cuales producen graves efectos que afectan la salud del paciente. Es conocido que entre las alteraciones metabólicas que afectan al ser humano, la obesidad es sin lugar a dudas una de las más importantes, tanto desde el punto de vista de su frecuencia como de sus repercusiones sobre la salud; además de ser el problema nutricional (Moráis, A; Martínez, V.; Dalmau, J; Martínez, M; Peña, L; Varea, V, 2012) más importante en el mundo desarrollado, pues el $10 \%$ de su población es obesa. En Ecuador actualmente la diabetes mellitus tipo II es la primera causa de muerte. El objetivo general es determinar la relación existente entre Obesidad y Diabetes Mellitus Tipo 2 y los objetivos específicos son describir las causas que produce la obesidad, determinar de qué manera se desarrolla la Diabetes Mellitus tipo 2, realizar campañas de prevención de obesidad a travez de charlas, talleres, folletos ilustrativos a la población expuesta. Los resultados indicaron que toda persona que tenga obesidad y sobrepeso tiene un $60 \%$ de posibilidad que pueda padecer diabetes; y el $40 \%$ no. La discusión de los resultados obtenidos indica que la obesidad influye directamente en la producción de Diabetes Mellitus tipo II.

Palabras claves: Obesidad; Diabetes Mellitus Tipo II; problema nutricional; causas que produce la obesidad; prevención de obesidad. 
Josefina E. Ramírez-Amaya; Pedro L. Maldonado-Álava; Asdrubal M. Fabre-Parrales

\begin{abstract}
The objective of the present work is to determine the relationship between Obesity and Type II Diabetes Mellitus and to describe the causes of obesity. Which produce serious effects that affect the health of the patient. It is known that among the metabolic alterations that affect the human being, obesity is undoubtedly one of the most important, both from the point of view of its frequency and its repercussions on health; Besides being the most important nutritional problem in the developed world, because $10 \%$ of its population is obese. In Ecuador, type II diabetes mellitus is currently the leading cause of death. The general objective is to determine the relationship between Obesity and Type 2 Diabetes Mellitus and the specific objectives are to describe the causes of obesity, to determine how Diabetes Mellitus type 2 develops, to carry out obesity prevention campaigns through lectures, Workshops, illustrative brochures to the exposed population. The results indicated that anyone who is obese and overweight has a $60 \%$ chance that they may have diabetes; And $40 \%$ did not. The discussion of the results indicates that obesity directly influences the production of Type II Diabetes Mellitus.
\end{abstract}

Key words: Obesity; Type II Diabetes Mellitus; Nutritional problem; Causes of obesity; Prevention of obesity. 


\section{Resumo}

O objectivo deste estudo é determinar a relação entre a obesidade e diabetes mellitus tipo II e descrever as causas que produzem a obesidade. que produzem efeitos graves que afetam a saúde do paciente. Sabe-se que entre as alterações metabólicas que afetam os seres humanos, a obesidade é sem dúvida um dos mais importantes, tanto do ponto de vista da sua frequência e seu impacto na saúde; Além de ser o mais importante no problema de nutrição mundo desenvolvido, já que $10 \%$ de sua população é obesa. Equador atualmente em diabetes mellitus tipo II é a principal causa de morte. O objectivo geral é determinar a relação entre obesidade e diabetes mellitus tipo 2 e os objectivos específicos são para descrever as causas que provoca obesidade, determinar como Diabetes Mellitus Tipo 2, campanhas conversas travez de prevenção da obesidade desenvolve, workshops, folhetos ilustrativos para a população exposta. Os resultados indicaram que todas as pessoas que têm a obesidade eo excesso de peso têm $60 \%$ de chance de que ele pode diabetes; e $40 \%$ não o fazem. Discussão dos resultados indica que a obesidade influencia diretamente a produção de diabetes mellitus tipo II..

Palavras-chave: Obesidade; Diabetes mellitus tipo II; problema nutricional; causas que produzem a obesidade; a prevenção da obesidade.. 


\section{Introducción.}

Es conocido que entre las alteraciones metabólicas que afectan al ser humano, la obesidad es sin lugar a dudas una de las más importantes, tanto desde el punto de vista de su frecuencia como de sus repercusiones sobre la salud; además de ser el problema nutricional más importante en el mundo desarrollado, pues el $10 \%$ de su población es obesa. (García Ferrera, 1996)

Definida como un exceso de tejido adiposo que se manifiesta por un peso inadecuado, la obesidad es una condición que afecta a sujetos de todas las edades y sexos.

Además, la obesidad se asocia o favorece la aparición de una serie de enfermedades que ocupan los primeros lugares dentro de los cuadros de morbilidad y mortalidad de la población, como son la cardiopatía isquémica, la hipertensión arterial, la diabetes mellitus tipo II, las enfermedades cerebrovasculares y algunos tipos de cáncer, lo que hace que la morbilidad y la mortalidad del sujeto obeso sea superior a la del sujeto con normopeso.

Los cambios positivos en el nivel socioeconómico de la sociedad en general, han mejorado las condiciones laborales y de vida de los trabajadores, una disminución de la actividad física, lo que favorece el sedentarismo. La vida sedentaria disminuye el gasto energético y favorece la aparición de la obesidad. (García Ferrera, 1996)

Por otro lado, la diabetes mellitus es una enfermedad endocrina, metabólica, vascular, crónica, producida por una interacción variable de factores genéticos y ambientales, promotores de una constelación de anomalías bioquímicas y anatómicas, que afectan el metabolismo de los carbohidratos, las grasas y las proteínas. Afecta a personas de todas las edades, sin distinción de razas o nivel socioeconómico, y se considera un problema universal en aumento. (Pérez Rivero, 2002)

Aunque la frecuencia real de la enfermedad es difícil de asegurar, dados los diferentes criterios diagnósticos existentes, se plantea que cerca de 135 millones de personas a nivel mundial la padecen, lo que representa de 2 a 5 \% de la población. (Pérez Rivero, 2002)

La diabetes tipo 2 (DM2) se presenta a cualquier edad, generalmente sobre los 40 años; aumentando el riesgo con la obesidad, la falta de actividad física, dislipidemia, antecedentes 
familiares o de diabetes gestacional, hipertensión y la edad. Por tanto, se debe considerar la DM2 como el fenotipo final de problemas metabólicos crónicos asintomáticos que se pueden iniciar en edades tempranas y que en la mayoría de los casos se pueden evitar modificando el factor ambiental. (Navarrete B, 2012)

La prevalencia de diabetes es mayor en los países desarrollados, sin embargo, el aumento proporcional de ésta es mayor en los que están en vías de desarrollo, tendencia que continuará durante los próximos años. (Navarrete B, 2012)

En Ecuador las poblaciones urbanas tienen tasas de prevalencia dos veces mayores que las rurales. (Navarrete B, 2012)

Por todas las razones expuestas, ésta se considera en la actualidad como un grave problema de salud, si a esto añadimos su origen multifactorial y los pobres resultados obtenidos en muchas ocasiones con los diferentes tratamientos, podemos afirmar que lo más importante es la prevención y para ello es de gran valor la creación de hábitos alimentarios correctos y el cambio del estilo de vida de la población. Si tomamos en cuenta que nuestro sistema de salud cuenta a nivel de atención primaria con un eslabón fundamental que es el médico de la familia, estamos en condiciones de afirmar que podemos establecer las acciones de salud necesarias para su control y prevención. (García Ferrera, 1996)

Motivados por todo esto, nos decidimos a realizar este trabajo con el objetivo de determinar la relación entre la obesidad y la aparición de diabetes mellitus tipo II.

\section{Objetivos específicos}

- $\quad$ Describir las causas que produce la obesidad (León, R; Jiménez, B; López, A; Barrera, K, 2014)

- $\quad$ Determinar de qué manera se desarrolla la Diabetes Mellitus tipo 2

- Realizar campañas de prevención de obesidad (Castillo, O; Téllez, L; Simón, J; Rodríguez, G, 2013) a través de charlas, talleres, folletos ilustrativos a la población expuesta. 
Josefina E. Ramírez-Amaya; Pedro L. Maldonado-Álava; Asdrubal M. Fabre-Parrales

La investigación realizada, es del tipo exploratoria y bibliográfica utilizando libros, revistas, folletos, y páginas de Internet de la OMS en donde se recopilaron todas las informaciones necesarias de otros estudios realizados, para ampliar los conocimientos sobre el tema de la investigación.

Exploratoria Porque se examinó el tema de investigación "Relación entre la obesidad y la aparición de diabetes mellitus tipo II", Se exploraron áreas problemáticas como el desconocimiento sobre el valor nutricional de los alimentos que favorece la obesidad y tiene como consecuencia la enfermedad de diabetes mellitus tipo II",

\section{Resultados.}

Los resultados incluyeron 10 investigaciones (algunas de estas cuentan con varias poblaciones) que describen la CVRS de 8.908 personas, 6.841 con diabetes mellitus tipo 1, 941 diabetes mellitus tipo 2.835 sobrepeso y 291 con obesidad; los estudios se desarrollaron en siete países diferentes, con un promedio de edad de 13,3 años y un rango entre 9 y 18 años (tabla 1).

\begin{tabular}{|c|c|c|c|c|c|c|}
\hline Autor & Alio & Lugar & Enfermedad & Edad $X \pm D S$ & $n$ & $\begin{array}{r}\text { Sujtos } \\
\text { \&i(C95\%) }\end{array}$ \\
\hline Filliard ME (21) & 2013 & USA & $\begin{array}{l}\text { Dibetes tipo I } \\
\text { Dibeten tipo? }\end{array}$ & $\begin{array}{l}12,1(4,7) \\
16,2(2,8)\end{array}$ & $\begin{array}{l}4.000 \\
548\end{array}$ & $\begin{array}{r}44,9(43,9 \cdot 45,9) \\
62.2(5,6 \cdot 6,7)\end{array}$ \\
\hline Naughton W (22) & 2008 & USA & $\begin{array}{l}\text { Dibten tipo } 1 \\
\text { Dibeten tipo? }\end{array}$ & $\begin{array}{l}14.6(3,6) \\
17,5(2.8)\end{array}$ & $\begin{array}{l}2188 \\
257\end{array}$ & $\begin{array}{r}24,6(23,7 \cdot 25,5) \\
29(29 \cdot 3.32)\end{array}$ \\
\hline Keting C. (23) & 2011 & Australia & $\begin{array}{l}\text { Sobbepses } \\
\text { Obeso }\end{array}$ & $14.6(1,4)$ & $\begin{array}{l}583 \\
181\end{array}$ & $\begin{array}{l}6,5(6,0-7,1) \\
20(1,7 \cdot 2,3)\end{array}$ \\
\hline Ostbye T (15) & 2010 & Singepour & $\begin{array}{l}\text { Sobrepeso } \\
\text { Obeso }\end{array}$ & $13,8(1,4)$ & $\begin{array}{l}195 \\
93\end{array}$ & $\begin{array}{l}2,2(1,9-2.5) \\
1,0(0,8-1,3)\end{array}$ \\
\hline Abdu|-Rasoul M (24) & 2013 & Kuwat & Dibeters tipo 1 & $91.13 .7)$ & 436 & $49(4,4 \cdot 5,3)$ \\
\hline Kalywa E (13) & 2011 & Grecid & Dibbetes tipo 1 & $10,91(3,99)$ & 128 & $1,4(1,2 \cdot 1,7)$ \\
\hline Etrinn T. Rhodes (25) & 2012 & USA & Dibbetes tipo? & $15,5(2,0)$ & 108 & $1,2(1,0 \cdot 1,4)$ \\
\hline Emmanoulidou \& (26) & 2008 & Grecit & Dibperestipo 1 & $10,97(3,79)$ & 89 & $10(0,8 \cdot 1,2)$ \\
\hline Nidia Kunke (14) & 2008 & Bnasi & $\begin{array}{l}\text { Sobrepses } \\
\text { Obero }\end{array}$ & $159,90,89)$ & $\begin{array}{l}57 \\
17\end{array}$ & $\begin{array}{l}0,6(0,5 \cdot 0.8) \\
0.2(0.1 \cdot 0.3)\end{array}$ \\
\hline Allan CL. 2.27) & 2008 & Candá & Diabtes tipo ? & $15(1,8)$ & 28 & $0.3(0,2 \cdot 0.4)$ \\
\hline TOTAL. & & & & 13.399-18) & 8908 & \\
\hline
\end{tabular}

Tabla 1.- Descripción de los estudios. 
Durante un período determinado de estudio se diagnosticaron y comenzaron tratamiento insulínico 50 niños y adolescentes que se han incluido en el análisis. Veinticuatro fueron mujeres (48\%) y veintiséis varones. La edad media al comienzo de la enfermedad fue de 10.5 años con un rango de 0.7 a 18.3 años y una mediana de 11.0 años. Presentaron antecedentes personales de riesgo para enfermedad cardiometabólica 20 pacientes (40.8\%), 19 (38\%) SP u OB al inicio de la diabetes o inmediatamente antes de la aparición de los síntomas atribuibles a la DM y 1 paciente $(2.2 \%)$ dislipidemia, mientras 4 pacientes $(8.3 \%)$ tenían enfermedad autoinmune pre-existente al comienzo de la DM (8.3\%). En relación a la forma de comienzo, todos refirieron el antecedente de pérdida de peso con poliuria y polidipsia en el mes previo al diagnóstico. Veintiuno (42\%) presentaron cetoacidosis como comienzo de su DBT, no encontrándose diferencias en la forma de presentación entre los pacientes con SP/ OB y aquellos con peso normal.

\begin{tabular}{|c|c|c|c|}
\hline & $\begin{array}{l}\text { Pacientes diabéticos } \\
\text { con sobrepeso/obesidad } \\
\qquad \mathrm{N}=19\end{array}$ & $\begin{array}{l}\text { Pacientes diabéticos } \\
\text { con normopeso } \\
\mathrm{N}=31\end{array}$ & $\mathrm{p}$ \\
\hline $\begin{array}{l}\text { Edad (años) } \\
\quad \text { Media } \pm \text { DE }\end{array}$ & $10.9 \pm 2.9$ & $10.3 \pm 4.3$ & $\mathrm{n} / \mathrm{s}$ \\
\hline $\begin{array}{l}\text { Score } Z \text { IMC } \\
\quad \text { Media } \pm \text { DE }\end{array}$ & $0.8 \pm 0.9$ & $-0.16 \pm 0.1$ & 0.01 \\
\hline Masa grasa (\%) & 21.7 & 14.7 & 0.002 \\
\hline $\begin{array}{l}\text { Glucemia basal (mg\%) } \\
\quad \text { Media } \pm \mathrm{DE}\end{array}$ & $128.26 \pm 40.7$ & $145.9 \pm 61.2$ & $\mathrm{n} / \mathrm{s}$ \\
\hline $\begin{array}{l}\text { Péptido } C \text { basal (ng/ml) } \\
\qquad \text { Media } \pm D E\end{array}$ & $2.3 \pm 2.6$ & $1.28 \pm 1.4$ & $\mathrm{n} / \mathrm{s}$ \\
\hline $\begin{array}{l}\text { Indice } \\
\text { Gluc/péptido C }\end{array}$ & 147.9 & 295 & $\mathrm{n} / \mathrm{s}$ \\
\hline $\begin{array}{l}\text { Antecedentes familiares } \\
\text { Sindrome metabólico }\end{array}$ & $17(89.5 \%)$ & $25(80.4 \%)$ & $n / s$ \\
\hline $\begin{array}{l}\text { Antecedentes familiares } \\
\text { Dislipidemia }\end{array}$ & $7(36.8 \%)$ & $10(32.3 \%)$ & $\mathrm{n} / \mathrm{s}$ \\
\hline $\begin{array}{l}\text { Antecedentes familiares } \\
\text { Obesidad }\end{array}$ & $14(73.7 \%)$ & $17(54.8 \%)$ & 0.09 \\
\hline $\begin{array}{l}\text { Antecedentes familiares } \\
\quad \text { Diabetes } 2\end{array}$ & $12(63.2 \%)$ & $11(35.5 \%)$ & 0.05 \\
\hline $\begin{array}{l}\text { Antecedentes familiares } \\
\text { Hipertensión }\end{array}$ & $9(50 \%)$ & $15(48.8 \%)$ & $n / s$ \\
\hline
\end{tabular}

Tabla 2.- Evaluación de factores de riesgo metabólico en pacientes diabéticos agrupados según su estado nutricional 


\section{Conclusiones.}

Se ha concluido en la presente investigación que toda persona que tenga obesidad y sobrepeso tiene un $60 \%$ de posibilidad que pueda padecer diabetes; en caso contrario el $40 \%$ no la padece.

A partir de la siguiente problemática, hemos estudiado y aprendido una de las formas más usuales del: cómo se llega a padecer la diabetes mellitus II a través de un problema muy común como lo es la obesidad que cada día incrementa más su efecto en la población mundial, esta relación se adapta y aborda un importante proceso de aprendizaje, destacando su biología, anatomía, nutrición y la función de cada uno de sus componentes.

Retomando los aspectos estudiados en este trabajo podemos concluir que la obesidad si no se controla a tiempo, se convierte en el camino más directo hacia la diabetes mellitus tipo II, sabiendo que la obesidad es una enfermedad crónica tratable que aparece cuando existe un exceso de tejido adiposo (grasa) en el cuerpo y que la diabetes tipo 2 es una enfermedad que dura toda la vida (crónica) en la cual hay un alto nivel de azúcar (glucosa) en la sangre.

Todo lo abordado nos da una pauta del trabajo que se requiere para tener un aprendizaje, capaz de comprender y dar soluciones a situaciones que favorezcan la calidad de vida. Como lo es en este caso obesidad y diabetes mellitus II que se han convertido en las enfermedades más mortíferas en los últimos tiempos. Por lo cual la prevención se toma como la mejor solución a los problemas de salud ya indicados. En cambio, hay factores que vienen del medio que serán más flexibles como la alimentación y el ejercicio.

\section{Bibliografía.}

García Ferrera, Waldo Orlando, Rodríguez de Miranda, Adelaida, Escobar Capote, María del Pilar, Haedo Castro, Dora, \& Medina González, Teresa. (1996). La obesidad como problema de salud en la comunidad. Revista Cubana de Medicina General Integral, 12(4), 335-341.

Navarrete B, Claudia, \& Cartes-Velásquez, Ricardo. (2012). Prevalencia de diabetes tipo 2 y obesidad en comunidades Pehuenches, Alto Biobio. Revista chilena de nutrición, 39(3), 7-10. https://dx.doi.org/10.4067/S0717-75182012000300001

Pérez Rivero, Jorge Luis, Regueira Naranjo, Jesús Lázaro, \& Hernández Hernández, Roberto. (2002). Caracterización de la diabetes mellitus en un área de salud. Revista Cubana de Medicina General Integral, 18(4), 251-253. 
Castillo, O; Téllez, L; Simón, J; Rodríguez, G. (2013). Factores que influyen en la prevención y tratamiento efectivo de la obesidad infantil. Investigación y Ciencia.

León, R; Jiménez, B; López, A; Barrera, K. (2014). Ideas sobre las causas de la obesidad en estudiantes universitarios mexicanos y españoles. Enseñanza e Investigación en Psicología.

Moráis, A; Martínez, V.; Dalmau, J; Martínez, M; Peña, L; Varea, V. (2012). Problemas nutricionales percibidos por los pediatras en niños españoles menores de 3 años. Nutrición Hospitalaria.

Moreno, D. M. (2012). Definición y clasificación de obesidad. Revista Médica Clínica Las Condes. 23(2). 124-125.

Ricardo, Y. R. (2012). Antropometría en el diagnóstico de pacientes obesos; una revisión. Madrid: Nutrición Hospitalaria.

Soca, P. E., \& Peña, A. N. (2009). Consecuencias de la obesidad. La Habana: ACIMED. 